\title{
Local Blockade of Sodium Channels by Tetrodotoxin Ameliorates Tissue Loss and Long-Term Functional Deficits Resulting from Experimental Spinal Cord Injury
}

\author{
Yang Dong Teng and Jean R. Wrathall \\ Department of Cell Biology, Neurobiology Division, Georgetown University, Washington, D.C. 20007
}

\begin{abstract}
Although relatively little is known of the mechanisms involved in secondary axonal loss after spinal cord injury (SCI), recent data from in vitro models of white matter (WM) injury have implicated abnormal sodium influx as a key event. We hypothesized that blockade of sodium channels after $\mathrm{SCl}$ would reduce WM loss and long-term functional deficits. To test this hypothesis, a sufficient and safe dose $(0.15 \mathrm{nmol})$ of the potent $\mathrm{Na}^{+}$channel blocker tetrodotoxin (TTX) was determined through a doseresponse study. We microinjected TTX or vehicle (VEH) into the injury site at 15 min after a standardized contusive SCI in the rat. Behavioral tests were performed $1 \mathrm{~d}$ after injury and weekly thereafter. Quantitative histopathology at 8 weeks postinjury showed that TTX treatment significantly reduced tissue loss at the injury site, with greater effect on sparing of WM than gray
\end{abstract}

matter. TTX did not change the pattern of chronic histopathology typical of this $\mathrm{SCl}$ model, but restricted its extent, tripled the area of residual WM at the epicenter, and reduced the average length of the lesions. Serotonin immunoreactivity caudal to the epicenter, a marker for descending motor control axons, was nearly threefold that of $\mathrm{VEH}$ controls. The increase in WM at the epicenter was significantly correlated with the decrease in functional deficits. The TTX group exhibited a significantly enhanced recovery of coordinated hindlimb functions, more normal hindlimb reflexes, and earlier establishment of a reflex bladder. The results demonstrate that $\mathrm{Na}^{+}$channels play a critical role in WM loss in vivo after SCl.

Key words: rat; spinal cord injury; sodium channel; tetrodotoxin; white matter injury; motor function; histopathology
The deleterious consequences of traumatic spinal cord injury (SCI) are largely attributable to disruption of descending and ascending white matter (WM) tracts. Tissue degeneration after SCI develops later and more slowly in WM than in gray matter (GM) (Lampert, 1967; Dohrmann et al., 1972; Balentine, 1978a,b; Ransom et al., 1990a). Furthermore, the sparing of even a small percentage of axons can significantly enhance function (Windle et al., 1958; Guth et al., 1980; Blight, 1983; Blight and Young, 1990). Thus, therapeutic interventions aimed at preservation of WM could be extremely important for SCI. The development of effective treatments, however, has been hampered by lack of knowledge of the mechanisms underlying secondary loss of WM after SCI. The importance of $\mathrm{Ca}^{2+}$ influx in axonal injury consequent to spinal cord trauma has long been postulated (Balentine and Spector, 1977). Nonetheless, the events leading to this abnormal $\mathrm{Ca}^{2+}$ influx have been obscure. Recent studies in vitro have identified some key events in WM injury that may also operate in vivo.

A preparation of rat optic nerve has been used to study the effects of anoxia in vitro (Ransom et al., 1990b; Stys et al., 1990, 1991a). The results indicate that $\mathrm{Ca}^{2+}$ influx into axons leads to irreversible damage. The abnormal influx of $\mathrm{Ca}^{2+}$ is mediated primarily by "reverse operation" of the $\mathrm{Na}^{+}-\mathrm{Ca}^{2+}$ exchanger (Stys et al., 1991b). This, in turn, is driven by abnormally high $\left[\mathrm{Na}^{+}\right]_{\mathrm{i}}$ and depolarization. $\mathrm{Na}^{+}$influx through $\mathrm{Na}^{+}$channels, under conditions of anoxia-

Received Jan. 21, 1997; revised March 20, 1997; accepted March 25, 1997.

This work was supported by National Institutes of Health Grants PO1-NS-28130 and RO1-NS-35647. We thank Ms. Sadia Aden and Dr. Chen-Xu Wang for their assistance in the behavioral evaluation of the animals.

Correspondence should be addressed to Dr. Jean R. Wrathall, Department of Cell Biology, Neurobiology Division, Georgetown University, 3900 Reservoir Road NW, Washington, DC 20007-2197.

Copyright (C) 1997 Society for Neuroscience $0270-6474 / 97 / 174359-08 \$ 05.00 / 0$ mediated dysfunction of $\mathrm{Na}^{+}-\mathrm{K}^{+}$ATPase, produces the accumulation of $\left[\mathrm{Na}^{+}\right]_{\mathrm{i}}$. Thus, blockers of $\mathrm{Na}^{+}$channels, such as tetrodotoxin (TTX), local anesthetics (Stys et al., 1992a,b), antiarrhythmics, and certain anticonvulsants (Stys, 1995; Fern et al., 1993), as well as antagonists of the $\mathrm{Na}^{+}-\mathrm{Ca}^{2+}$ exchanger, e.g., benzamil and bepridil (Stys et al., 1992b), are highly protective against anoxic injury in this in vitro model.

In addition, mechanical injury in the absence of anoxia was studied in an in vitro preparation of rat spinal dorsal funicular WM (Agrawal and Fehlings, 1996). The results indicate that axonal injury attributable to compression can also be mitigated by TTX. Instead of benzamil and bepridil, blockers of the $\mathrm{Na}^{+}-\mathrm{H}^{+}$exchanger (amiloride and harmaline) were significantly protective, suggesting a link between increased $\left[\mathrm{Na}^{+}\right]_{\mathrm{i}}$ and intra-axonal acidosis.

Therefore, $\mathrm{Na}^{+}$influx through axonal $\mathrm{Na}^{+}$channels is implicated in secondary injury of WM after both anoxia and mechanical compression. Because both compression and ischemia/anoxia occur in traumatic SCI (Tator and Fehlings, 1991; Young, 1993), it is logical to postulate that similar mechanisms operate acutely in vivo and could contribute to secondary WM loss. We hypothesized that $\mathrm{Na}^{+}$influx is one of the key elements leading to WM degeneration after SCI.

To test this hypothesis, we microinjected TTX, one of the most potent $\mathrm{Na}^{+}$channel blockers known (Hille, 1992), into the injury site beginning at 15 min after a standardized spinal cord contusion injury in the rat. We determined the effects of TTX on functional deficits over time after injury, and on quantitative histopathology at 8 weeks after SCI.

\section{MATERIALS AND METHODS}

SCI. Female Sprague Dawley rats (230-250 gm) were anesthetized with chloral hydrate $(360 \mathrm{mg} / \mathrm{kg}$, i.p.). A laminectomy at the T8 vertebral level 
exposed a 2.8-mm-diameter circle of dura, and the animals were then stabilized with Allis clamps on the T7 and T9 spinous processes. The impounder tip of a weight-drop device was rested on the dura, and the contusion injury was produced by dropping a $10 \mathrm{gm}$ weight from the height of $2.5 \mathrm{~cm}$ onto the impounder (Wrathall et al., 1985, 1994; Teng and Wrathall, 1996). This model has been characterized in terms of biomechanics (Panjabi and Wrathall, 1988), resulting functional deficits (Gale et al., 1985; Wrathall et al., 1985), somatosensory evoked potentials (Raines et al., 1988), effects on the blood-spinal cord barrier (Noble and Wrathall, 1989b), and quantitative histopathology (Noble and Wrathall, 1985, 1989a).

After SCI, manual expression of bladders was performed twice daily until a reflex bladder was established, which usually happens in the second week postoperatively. Postinjury (p.i.) care also included housing the rats in pairs to reduce isolation-induced stress, maintaining ambient temperature at $22-25^{\circ} \mathrm{C}$, and using highly absorbent bedding. No prophylactic antibiotics were given.

TTX administration. Dose-response studies were used to determine a dosage regimen for TTX (RBI, Natick, MA) that would block $\mathrm{Na}^{+}$channels for a substantial period of time without significant mortality. TTX was dissolved in citrate buffer $(1.5 \mathrm{~mm}, \mathrm{pH} 4.8)$ at a concentration of 300 or 500 $\mu \mathrm{M}$ and a total dose from 0.15 to $1 \mathrm{nmol}$, in volumes ranging from 0.5 to 2 $\mu \mathrm{l}$, and was microinjected into the spinal cord through a stereotaxically placed 33 gauge needle inserted into the spinal cord $1 \mathrm{~mm}$ below the dura. The needle was linked with a $10 \mu \mathrm{l}$ microsyringe (Hamilton, Reno, NV) through polyethylene (PE 10) tubing (Clay Adams, Parsippany, NJ). The microsyringe was expressed, at rates from $0.1 \mu \mathrm{l}$ to $0.2 \mu \mathrm{l} / \mathrm{min}$, with a syringe pump (Model 341A, Sage Instruments, Cambridge, MA).

On the basis of the results from these preliminary studies, in the definitive experiment TTX was dissolved in citrate buffer at a concentration of $300 \mu \mathrm{M}$. Vehicle (VEH)-treated control animals received citrate buffer solution alone. Solutions were sterilized through $0.22 \mu \mathrm{m}$ filters (Millipore, Bedford, MA, USA). A total volume of $0.5 \mu$ l of TTX $(0.15$ nmol) or VEH was injected into the injury site over a period of $5 \mathrm{~min}$. After the injection, the needle was kept in the spinal cord for an additional $2 \mathrm{~min}$ to reduce the possibility of losing injected solution from the site.

Experimental protocol. The experiments were performed according to a randomized block design. Experimental group size was decided on the basis of power analysis of outcome measure data from injury doseresponse studies using this SCI model (Gale et al., 1985; Noble and Wrathall, 1985; Wrathall et al., 1985). On the basis of these analyses, with 12 rats per group there is an $80 \%$ probability of detecting an effect $\geq 34 \%$ in inclined plane score, $25 \%$ in the combined behavioral score, and $15 \%$ in WM area at the epicenter (see description of these outcome measures below). The TTX-treated and VEH-treated control groups $(n=12$ per group) were behaviorally tested at $1 \mathrm{~d}$ and weekly thereafter through 8 weeks after injury. The animals were then reanesthetized, and the spinal cord tissue was fixed by perfusion for histopathological analyses, as described below.

Behavioral evaluations of functional deficits. Tests of functional deficits were performed by one individual blind to the treatments, and the results were confirmed by separate evaluations of the rats by a second independent and blinded investigator. At each time point a battery of tests of hindlimb reflexes as well as coordinated use of hindlimbs were used, as described previously (Gale et al., 1985; Kerasidis et al., 1987). The reflexes tested included toe spread, placing, withdrawal in response to extension, pressure or brief pain, righting, and the reflex to lick the toes in response to heat. Coordinated motor activity that was assessed included open-field locomotion, swimming, and ability to maintain position on an inclined plane. Results in individual tests were examined separately, and in addition, overall hindlimb impairment was estimated with a combined behavioral score (CBS) that ranges from 0 (normal rat) to 100 (rat with no evidence of hindlimb function). The CBS was developed on the basis of initial injury dose-response studies (Gale et al., 1985). It exhibits a normal distribution, as formally tested with the Wilk-Shapiro procedure (Shapiro and Wilk, 1965), and was designed as a parametric statistic to provide a continuous measure of overall hindlimb deficits that is correlated to injury severity. It has greater statistical power than any of its component behavioral tests (Gale et al., 1985) and is significantly correlated with both degree of initial mechanical injury (Panjabi and Wrathall, 1988) and chronic histopathology (Noble and Wrathall, 1985, 1989a).

In addition, a more detailed examination of open-field locomotion was performed using an expanded scale that ranges from 0 to 21 , where 0 reflects no locomotory function and 21 reflects a normal performance (Basso et al., 1995). This "BBB" scale has been adopted by the Multicenter Animal Spinal Cord Injury Study, which is currently engaged in preclinical screening of potential therapeutic agents for SCI. Therefore, use of the BBB as an outcome measure after experimental SCI supports easier interlaboratory comparison of results.

Histopathology. After the 8 week behavioral testing, animals were anesthetized with chloral hydrate and perfused intracardially with saline followed by $4 \%$ paraformaldehyde in PBS, $\mathrm{pH}$ 7.4. Spinal cord tissue was removed from the vertebral canal, and a $1.5 \mathrm{~cm}$ segment centered at the injury site was left in fixative for an additional hour, equilibrated with increasing concentrations of sucrose solutions $(10-20 \%)$, and frozen with dry ice-isopentane $\left(-50^{\circ} \mathrm{C}\right)$. Twelve spinal cords in each group were sectioned for morphometric and immunocytochemical analyses. Serial 20 $\mu \mathrm{m}$ cross sections were cut with a Jung Frigicut $2800 \mathrm{E}$ cryostat, mounted with five sections $(100 \mu \mathrm{m}$ of tissue) per slide on slides that were coated with 3-aminopropyltriethoxysilane (Koo et al., 1988). All morphological analyses were performed with tissue identified only by animal number: the evaluator was blind to the treatment group until after the primary data were collected.

For morphometry, every tenth slide was stained with luxol-blue/hematoxylin and eosin, and projected. Areas of GM, myelinated WM, hypomyelinated WM, lesion cells, and cavities were traced as described previously (Noble and Wrathall, 1985). The tracings were digitized, and areas of each tissue component were calculated with a Zeiss IBAS image analysis system, through which three-dimensional reconstructions of the lesions were also generated.

5-HT immunoreactivity was used as a marker for descending serotonergic innervation from the brainstem (Skagerberg and Bjorklund, 1985; Tork, 1990). Rabbit antibody to 5-HT conjugated to bovine serum albumin (BSA) with paraformaldehyde was purchased from Incstar (Minneapolis, MN; catalog no. 20080). The antibody was purified with the aid of a BSA-agarose (Sigma, St. Louis, MO; catalog no. A-3790) column, to preclude nonspecific binding to BSA, and used at a dilution of 1:2000. Spinal cord sections were selected that represented $3 \mathrm{~mm}$ rostral and caudal to the lesion epicenter. All of the selected sections were processed together for immunocytochemistry, using the same solutions to allow comparison of the relative staining for 5-HT. Using a Zeiss IBAS image analysis system, fields of view containing the ventral horns were analyzed to determine the total areas (pixels) of stained immunoreactive terminals. The same processing parameters were used for all samples. The data were combined to estimate total immunoreactivity $\left(\mu \mathrm{m}^{2}\right)$ for the ventral horn area.

Statistical analyses. Because both CBS and inclined plane data represent parametric data as well as repeated measures, the data were analyzed statistically using repeated measures ANOVA, followed by Tukey's test for multiple comparisons between groups. The appropriateness of the model, initially recommended by Dr. D. Mundt (Division of Biostatistics and Epidemiology, Georgetown University) and used in previous studies (e.g., Wrathall et al., 1992, 1994), was tested with each individual set of data using the SAS statistical software. Nonparametric data (i.e., motor scores) were compared with the Wilcoxon test. BBB scores were also analyzed by ANOVA with repeated measures (Basso et al., 1995), followed by Tukey's test for differences at individual time points. For comparison of areas of spared tissue at different levels of the spinal cord rostral and caudal to the epicenter in TTX and VEH groups, repeated measures ANOVA was used, followed by Tukey's test for differences at individual levels of spinal cord. For comparison of 5-HT immunoreactive terminal area, the unpaired Student's $t$ test was used. All values are expressed as mean \pm SEM. Use of the term "significant" in the text indicates that statistical testing was performed and $p<0.05$.

\section{RESULTS}

\section{Feasible dose of TTX to block sodium channels in thoracic spinal cord}

Considering the well defined pharmacological nature as well as the potent toxicity of TTX, we conducted a step-by-step doseresponse study to identify a feasible dose of TTX for testing our hypothesis. We began the experiments by using $1 \mathrm{nmol}$ TTX, with $2 \mu \mathrm{l}$ of a $500 \mu \mathrm{M}$ solution infused at a rate of $0.2 \mu \mathrm{l} / \mathrm{min}$ into the injury site beginning at $15 \mathrm{~min}$ after SCI. This dose is twice the amount of TTX used to block axonal activity in the cortex of rat for an electrophysiological observation period of $6 \mathrm{hr}$ (Carmi- 


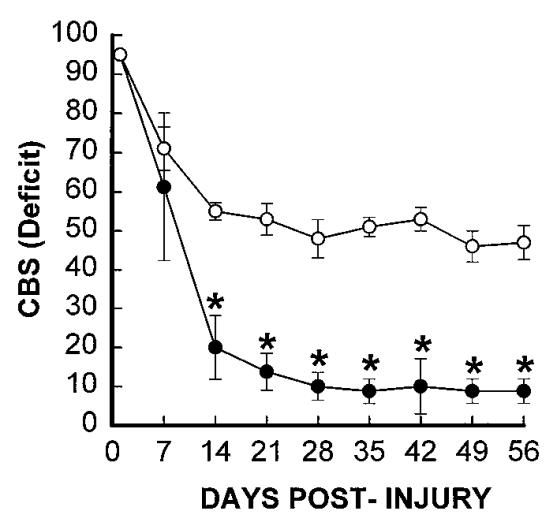

Figure 1. Effect of TTX on overall hindlimb deficits over time after SCI. Deficits are expressed as a combined behavioral score (CBS) (Gale et al., 1985). Data points represent the average CBS per group where groups received either VEH alone $(\bigcirc, n=4)$ or $1.0 \operatorname{nmol}$ TTX $(\bullet, n=5)$. Data were analyzed with repeated measures ANOVA, which showed an overall significant $(p<0.05)$ effect of treatment. Asterisks indicate that means are significantly different from the VEH-treated control group at the specified times after SCI (Tukey's procedure).

gnoto and Vicini, 1992). Fifty percent of the SCI rats given this dose of TTX (5 of 10) died of cardiorespiratory failure (confirmed by cardiorespiratory electrophysiology; data not shown) within 2 hr after the TTX injection. The surviving five rats, however, showed a dramatic reduction of functional deficits over an 8 week observation period as compared with a VEH-treated control group $(n=4)$ (Fig. 1). Histopathological analyses revealed that the TTX-treated group had a significantly reduced lesion length $(7.75 \pm 0.75$ vs $10.25 \pm 0.63$; unit: $\mathrm{mm})$, suggesting a reduction in secondary injury. Because of the high mortality rate, however, the apparent effect of TTX could be attributed to the preferential survival of relatively less injured animals.

We reasoned that the TTX, injected in the thoracic cord (T8), had probably reached more rostral spinal segments that are important in regulating cardiorespiratory function. Therefore, reductions in both the quantity of TTX and the administration volume seemed equally important to achieve a lower mortality rate. Thus, we performed a series of studies with different doses of TTX $(1.5,1.0,0.75$, or $0.5 \mu \mathrm{l}$ of either a 300 or $500 \mu \mathrm{M}$ TTX solution) under electrophysiological observation of cardiorespiratory function as well as behavioral evaluation of the effect of the drug in blocking axonal activity (i.e., causing hindlimb paralysis in otherwise normal rats). Injection of VEH solution alone produced no discernible effect: there were no behavioral abnormalities when rats $(n=4)$ were tested after recovery from anesthesia. We found that a $0.5 \mu \mathrm{l}$ volume of a $300 \mu \mathrm{M}$ TTX solution (total dose of $0.15 \mathrm{nmol}$, infused at the rate of $0.1 \mu \mathrm{l} / \mathrm{min}$ under general anesthesia) paralyzed the hindlimbs of normal rats for $43.8 \pm 3.3$ hr $(n=5)$ without causing any mortality. These rats recovered fully after the period of paralysis caused by TTX and thereafter exhibited no deficits in any of our behavioral tests. Another small preliminary group of rats given this dose at $15 \mathrm{~min}$ after SCI $(n=$ 3) also survived without mortality. Hence, this dose of TTX (0.15 nmol in $0.5 \mu \mathrm{l}$ ) was chosen for use in the definitive study reported below. With this dose, all rats ( $n=12$ per group) survived the full 8 week course of the experiment without evidence of any chronic adverse physical conditions other than those commonly associated with SCI.

\section{Effects of TTX on functional deficits after SCI}

As described previously by our laboratory (Gale et al., 1985; Noble and Wrathall, 1989a), rats demonstrated profound impairment of hindlimb function at $1 \mathrm{~d}$ after SCI, including areflexia and lack of coordinated motor functions such as locomotion. Thereafter, partial recovery of function was seen until a plateau was reached at 3-4 weeks, reflecting the long-term deficits characteristic of this degree of SCI. Focal infusion of TTX dramatically increased the speed and extent of recovery of hindlimb reflexes after SCI, as exemplified by the withdrawal reflex to a pressure stimulus (Fig. 2A), and righting reflex (Fig. 2B) (other reflex data not shown). Recovery of coordinated motor functions including swimming (Fig. $3 A$ ) and maintaining body position on an inclined plane (Fig. $3 B$ ) were significantly enhanced. Recovery of capacity to use the hindlimbs in open-field locomotion, as graded by the motor score that ranges from 0 in completely paralyzed rats to 5 in normal animals, was also significantly improved at days 14, 21 , 28, and 56 p.i. (Fig. 4). Overall hindlimb functional deficits, as assessed by the CBS, were reduced significantly compared with

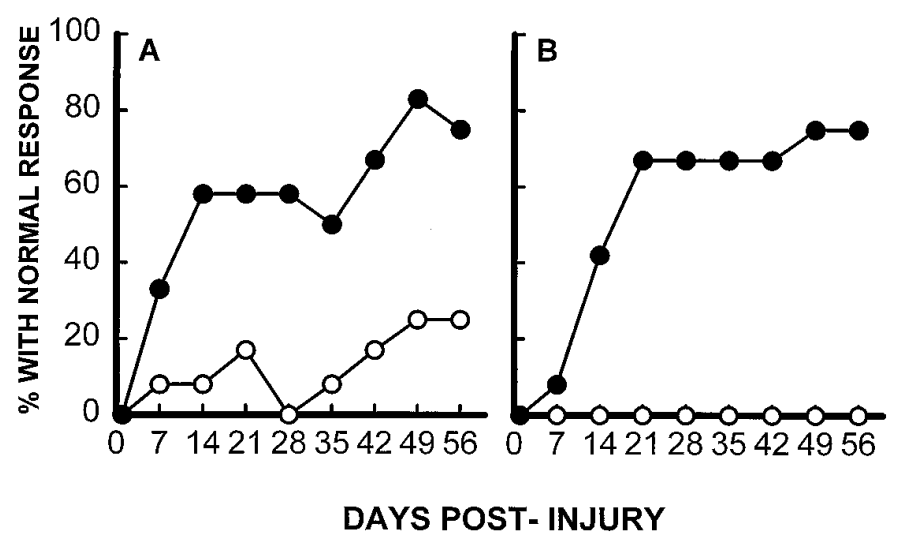

Figure 2. Effect of TTX $(0.15 \mathrm{nmol})$ on recovery of hindlimb reflexes. $A$, Effect of TTX on recovery of the reflex to withdraw the hindlimb in response to pressure applied to the toe pads (pressure withdrawal reflex). $B$, Effects of TTX on recovery of the righting reflex to turn over in response to abnormal body position (righting reflex). Data points represent the percentage of rats in each group $(n=12$ per group; VEH, $\bigcirc$; TTX, ๑) that exhibit a normal reflex.

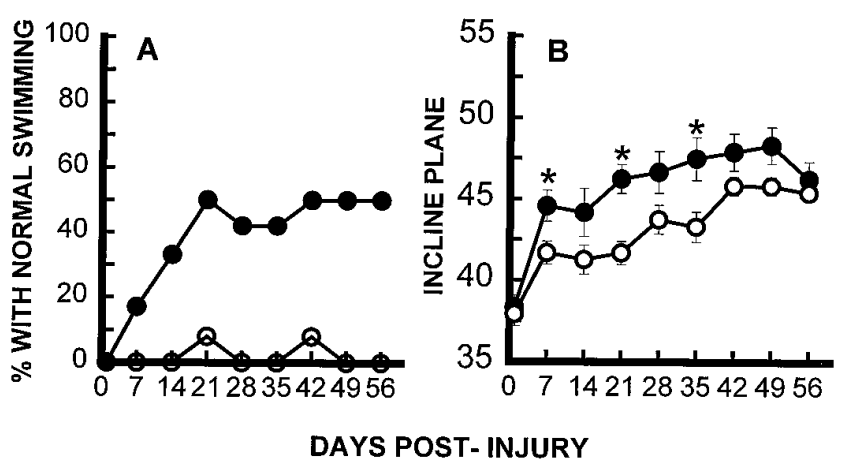

Figure 3. Effect of TTX on recovery of coordinated hindlimb functions. Comparison of groups $(n=12)$ that received VEH alone $(\bigcirc)$ or TTX $(\bullet)$. $A$, Percentage of rats that use their hindlimbs in swimming. $B$, Inclined plane performance. Data points represent average \pm SEM maximum angle at which rats can maintain position for $5 \mathrm{sec}$. Data were analyzed with repeated measures ANOVA, which showed an overall significant $(p<0.05)$ effect of treatment. Asterisks indicate that means are significantly different from the control group at the specified times after SCI (Tukey's procedure). 


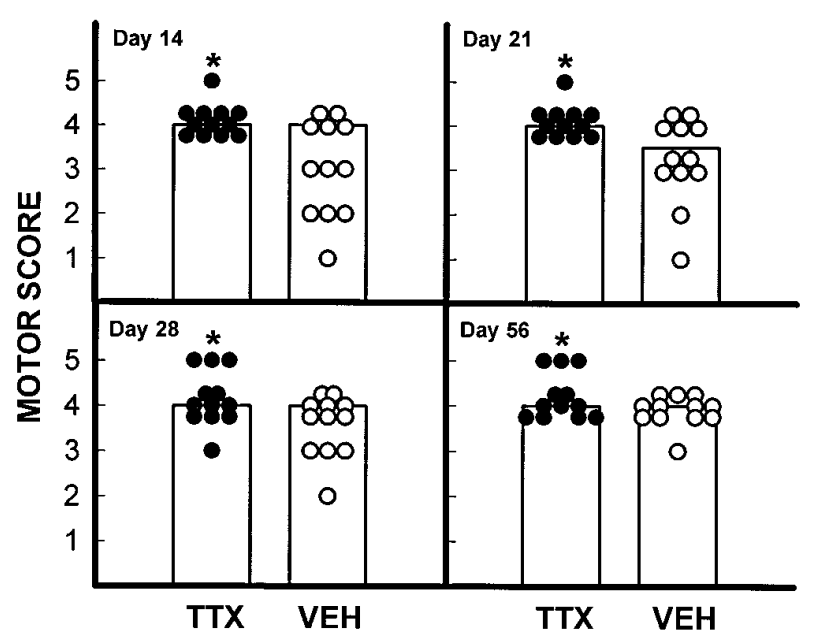

Figure 4. Effect of TTX on the recovery of locomotor function after SCI. Open-field locomotion was observed and graded on a $0-5$ scale (the motor score) (Wrathall et al., 1985), in which a grade of 3 or more indicates the ability to bear weight and use the hindlimbs for effective locomotion. Data points are individual scores for rats that received TTX $(\bullet)$ or VEH alone (O) $(n=12$ per group). The bars represent the modal score for the group. The extent of recovery as measured by the motor scores was significantly $(p<0.05)$ higher in the TTX group at $14,21,28$, and $56 \mathrm{~d}$ p.i. (Wilcoxon scores for rank sums).

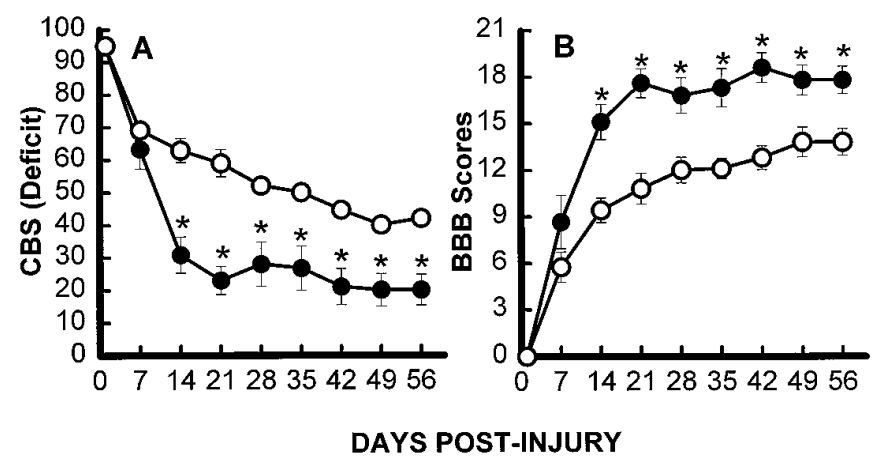

Figure 5. Hindlimb function over time after SCI in groups $(n=12)$ that received either VEH alone $(\bigcirc)$ or $0.15 \mathrm{nmol}$ TTX $(\bullet)$. $A$, Overall functional deficits expressed as the combined behavioral score $(C B S)$ (Gale et al., 1985). $B$, Locomotor function graded on an expanded scale $(B B B)$ (Basso et al., 1995) that ranges from 21 in normal rats to 0 in rats with complete hindlimb paralysis. Data points represent the group average. Where no error bar is shown, the SEM was smaller than the symbol. Data were analyzed with repeated measures ANOVA, which showed an overall significant $(p<0.05)$ effect of treatment. Asterisks indicate that means are significantly different from the VEH-treated control group at the specified times after SCI (Tukey's procedure).

the VEH controls beginning 2 weeks after SCI and throughout the 8 week course of the study (Fig. $5 A$ ). We also evaluated use of the hindlimbs in locomotion with the expanded BBB scoring system (Basso et al., 1995), which showed a significantly improved score in the TTX-treated group from 2 through 8 weeks after SCI (Fig. $5 B)$. This more detailed assessment of locomotor function demonstrated a more robust effect than that seen with the motor score (compare Figs. 4 and 5B).

After SCI, physiological micturation is lost, and manual expression of the bladder is required for the rats until a reflex bladder is established, usually in the second week after this degree of SCI (Wrathall et al., 1985). In addition to the effects on hindlimb functional deficits, focal application of TTX was also associated with a significant reduction in the number of days required to develop a reflex bladder $(5.08 \pm 0.53$ vs $7.50 \pm 0.26$; unit: day $)$.

\section{Effects of TTX on the histopathology produced by SCI}

As described previously for this model of SCI (Noble and Wrathall, 1985, 1989a), the lesioned area of the cord exhibited an elongated ovoid form with maximal tissue loss at the so-called "lesion epicenter." As exemplified by a three-dimensional reconstruction of the injury site from a rat typical of the VEH-treated group (Fig. 6D), the lesion tapered rostral and caudal to the epicenter, with its most distal elements ending in the ventral portion of the dorsal funicular WM. At the epicenters (Fig. 6C), the cross-sectional profile of the spinal cords from the VEHtreated group was reduced compared with the normal thoracic cord or the cross sections of the injured cord rostral and caudal to the epicenter. The epicenters were characterized by a peripheral, often incomplete, rim of residual WM, with the occasional presence of the most peripheral elements of GM. This residual rim surrounded the central lesion, consisting of cavities and a loose network of non-neuronal cells. There was a marked decrease in the extent of the lesions in most TTX-treated animals as shown in the reconstruction of the injury site of a rat typical of the TTX group (Fig. 6B). At the epicenters of lesions in the TTX group (Fig. $6 A$ ), there appeared to be more residual spinal cord tissues, especially WM. The cross sections showed profiles that frequently looked larger and closer to the diameter of the normal thoracic spinal cord.

The images of the spinal cord sections were analyzed further using morphometric techniques. Determinations of WM area in sections of the epicenter and at specified distances rostral and caudal to it demonstrated a significant increase in residual WM at the epicenter and at $1 \mathrm{~mm}$ rostral and 1 and $2 \mathrm{~mm}$ caudal to the epicenter (Fig. 7A). Quantitatively, the greatest effect of TTX was seen at the epicenter, which was also the injection site. At this site the average WM area was more than threefold that in the VEH control group. Although the results obtained from analysis of the GM area showed a similar trend, the differences were quantitatively less, and only significant at 2 and $3 \mathrm{~mm}$ caudal to the epicenter (Fig. 7B).

The relationship between WM sparing at the lesion epicenter and chronic hindlimb function at 8 weeks after SCI was examined. Linear regression analysis indicated a significant negative correlation $(r=0.796)$ between area of preserved WM and overall functional deficit, as measured by the CBS (Fig. 8).

Measurement of longitudinal lesion lengths also showed a significant effect of TTX. The average lesion length in the TTXtreated group was $6.50 \pm 0.54$ compared with $8.92 \pm 0.67$ (unit: $\mathrm{mm}$ ) in the VEH-treated control group. Treatment with TTX was associated with a $\sim 27 \%$ decrease in the length of the lesion.

To see whether focal application of TTX had altered not only the extent but also the typical pattern of chronic histopathology after SCI, we did sector analysis of the spared tissue from sections representing the lesion epicenters and $3 \mathrm{~mm}$ caudal to the epicenters. For WM analysis, cross-sectional profiles were divided into six radial sectors: the dorsal funiculus (Sector 1), left and right dorsal lateral funiculi (Sectors 2 and 6), left and right ventral lateral funiculi (Sectors 3 and 5), and ventral funiculus (Sector 4) (Fig. 9A). Sector analysis of WM at the epicenter (Fig. 9B) showed a generalized effect of TTX, with significant sparing in five of the sectors and a similar tendency in the sixth. Significant WM sparing at $3 \mathrm{~mm}$ caudal to the epicenter was, as expected, in the dorsal funicular WM (Sector 1), the area most likely to be lost 


\section{A}

$\operatorname{TTX}$
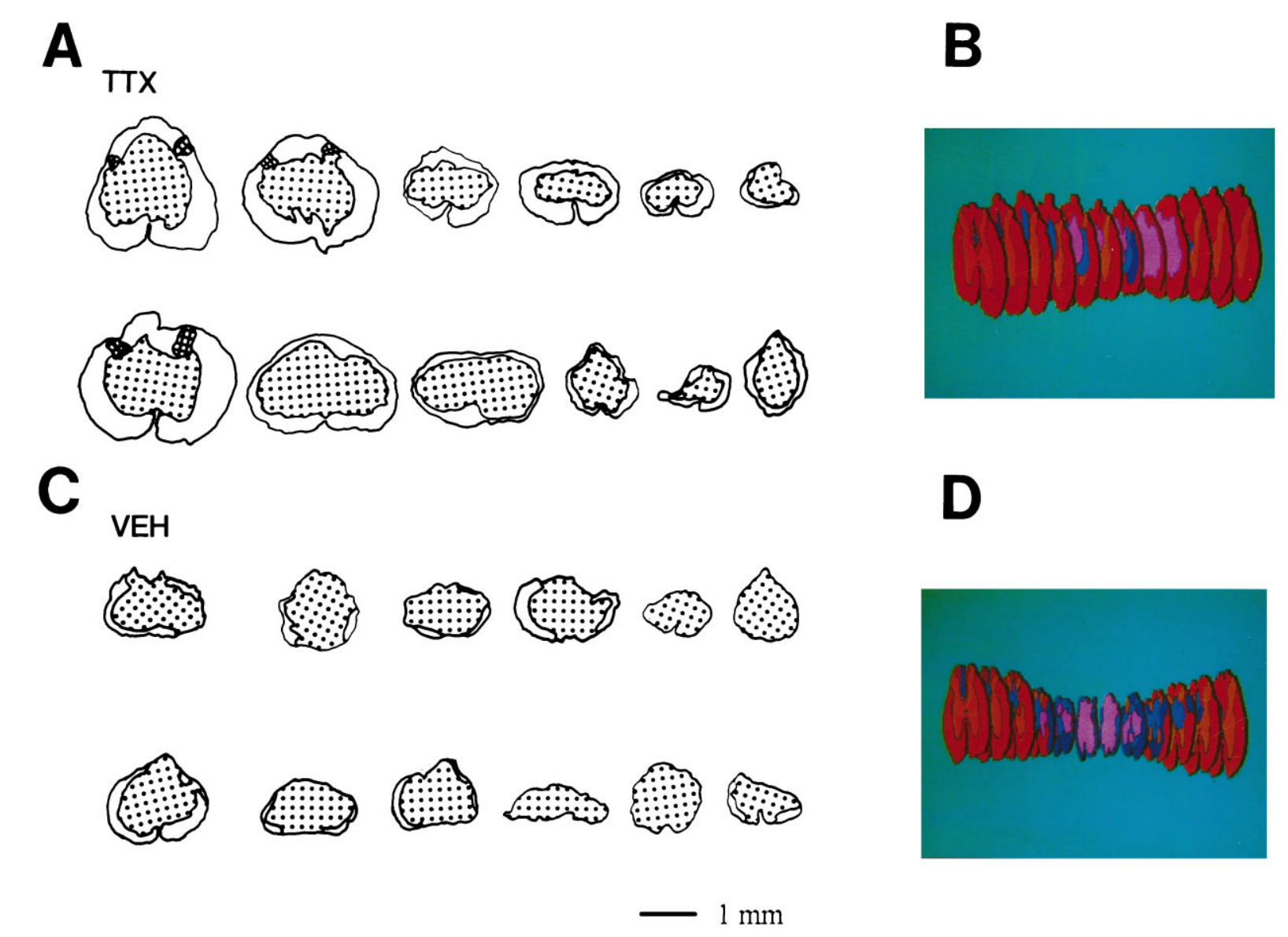

Figure 6. Effect of TTX on residual spinal cord tissue at 8 weeks after injury. Tracing of sections through the lesion epicenters in rats of the TTX-treated group $(A)$ and VEH-treated control group $(C)$. Epicenters from the TTX-treated group usually show a complete thin rim of peripheral myelinated and hypomyelinated WM (not distinguished in these tracings), and in occasional cases, peripheral portions of dorsal horn GM (cross-hatched). The centers of the lesions contain cavities and a loose network of lesion cells (stippled). Also shown are three-dimensional reconstructions of spinal cord of two individual rats representative (with final CBS similar to the group mean) of the TTX-treated group $(B)$ and the VEH-treated group $(D)$. Color code: red for WM; orange for GM; dark blue for hypomyelinated WM; blue for lesion cells; and pink for cavity.

after SCI in sections distal to the epicenter (Fig. 9C). There was no significant GM at the epicenter in either group, but at $3 \mathrm{~mm}$ caudal to the epicenter the effect of TTX was to spare GM sectors 2,4 , and 5, those closest to the dorsal funicular focus of the distal lesion and closest to the location of the WM spared in the TTX-treated group (Fig. 10A,B). Thus, TTX treatment did not change the pattern of chronic histopathology typical of this model of SCI; rather it restricted the extent of tissue loss.

Serotonin-immunoreactive terminals in the ventral horn areas were evaluated quantitatively in sections $3 \mathrm{~mm}$ rostral and caudal to the epicenter. The values in the rostral tissue sections were similar for the TTX-treated and VEH-treated groups $(859.26 \pm$ 36.83 vs $793.72 \pm 47.21$; unit: $\mu \mathrm{m}^{2}$ ); however, $3 \mathrm{~mm}$ caudal to the epicenter, the 5-HT terminal area averaged significantly higher in the TTX-treated group $(226.75 \pm 51.75$ vs $79.60 \pm 21.62$; unit: $\mu \mathrm{m}^{2}$ ), suggesting that the spared WM included axons of this important descending motor control pathway from the brain stem (Skagerberg and Bjorklund, 1985; Faden et al., 1988; Tork, 1990).

\section{DISCUSSION}

Our results demonstrate that the potent $\mathrm{Na}^{+}$channel blocker TTX applied focally at the injury site after SCI results in significant long-term tissue sparing and reduced functional deficits. At the time at which TTX was applied, $15 \mathrm{~min}$ p.i., the axonal

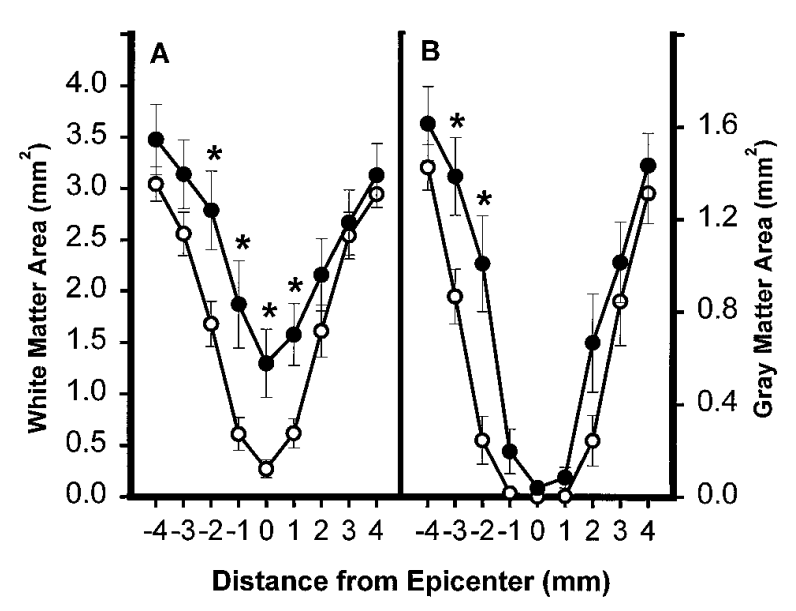

Figure 7. Effect of TTX on lesion morphometry at 2 months after injury. The area of spared WM $(A)$ and GM $(B)$ in spinal cord sections caudal $(-1$ to $-4 \mathrm{~mm})$ and rostral $(1-4 \mathrm{~mm})$ to the injury epicenter. Data represent the average from 12 rats per group. Groups received VEH alone $(\bigcirc)$ or $0.15 \mathrm{nmol}$ TTX $(\bullet)$. There was a significant overall difference $(p<$ 0.05 ) between the groups (repeated measures ANOVA). Asterisks indicate that means are significantly different from the VEH-treated group at the specified locations (Tukey's procedure). 


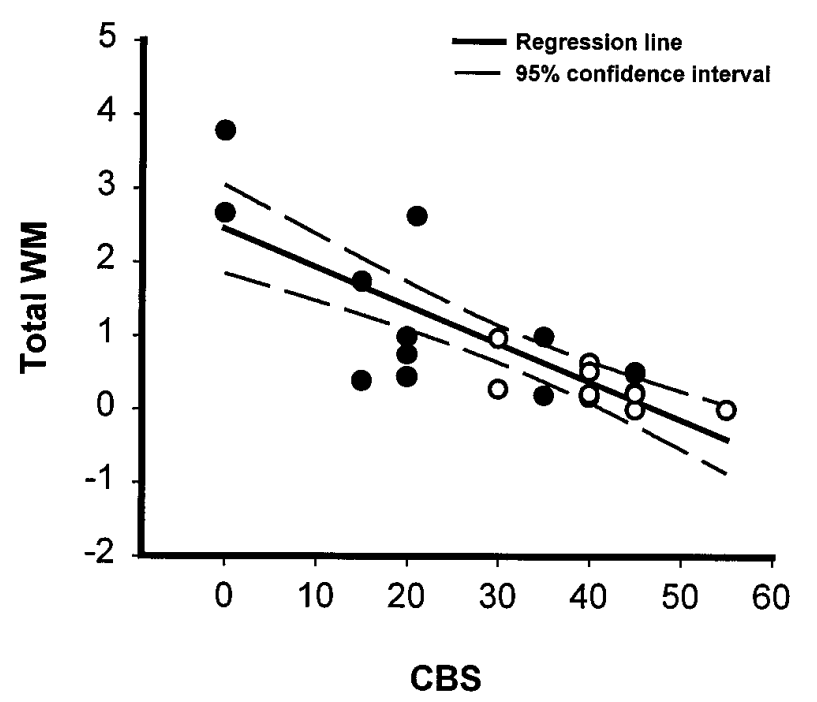

Figure 8. Linear regression analysis. A significant correlation between sparing of total WM (myelinated + hypomyelinated WM) at the lesion epicenter and reduction of functional deficits as estimated by the CBS at 8 weeks after SCI. $r=0.796 ; R^{2}=0.634 ; p<0.001$ (ANOVA). Analysis was based on 12 rats from each group that received VEH alone $(O)$ or 0.15 $\operatorname{nmol} \operatorname{TTX}(\bullet)$.
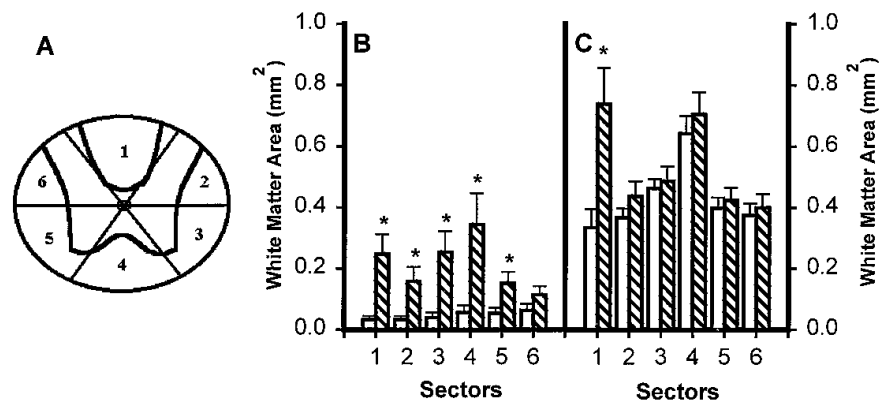

Figure 9. Effect of microinjected TTX on residual WM in different radial sectors of the spinal cord at 8 weeks after injury. $A$, WM sectors that were analyzed. $B$, Sector analysis of sections at the lesion epicenter in groups of rats microinjected with VEH alone (open bars) or $0.15 \mathrm{nmol}$ TTX (hatched bars $)$ at $15 \mathrm{~min}$ after SCI ( $n=12$ per group). $C$, Analysis of sectors at 3 $\mathrm{mm}$ caudal to the epicenter. Repeated measures ANOVA indicates an overall significant difference $(p<0.05)$. Asterisks signify that means are significantly different from the VEH-treated group in the specified sectors (Tukey's procedure)

pathology after SCI has not yet fully developed (Balentine, 1978a,b). The dose of TTX that we used, 0.15 nmol, blocked axonal conduction in normal rats for $\sim 43 \mathrm{hr}$, and thus was likely to be present in effective concentrations at the injury site during the critical period (i.e., $4 \mathrm{hr}$ p.i.) of development of acute axonal pathology after SCI (Lampert, 1967; Dohrmann et al., 1972; Balentine, 1978a,b; Rosenberg et al., 1996). The lengthy period $(43.8 \pm 3.3 \mathrm{hr} ; n=5)$ of hindlimb paralysis in normal animals with TTX injection, however, suggests that the conduction block may be attributable to more than the direct blockade of $\mathrm{Na}^{+}$channels at Nodes of Ranvier. In this respect, the report that TTX can be toxic to astrocytes in culture (Sontheimer et al., 1994) may be relevant. Because astrocytes are needed to maintain appropriate ionic conditions at the Nodes of Ranvier (Orkand, 1977; Newman, 1993), a possible TTX-mediated astrocytic toxicity in vivo could contribute to the long conduction block we observed. Current electron microscopic studies in our laboratory may shed light on
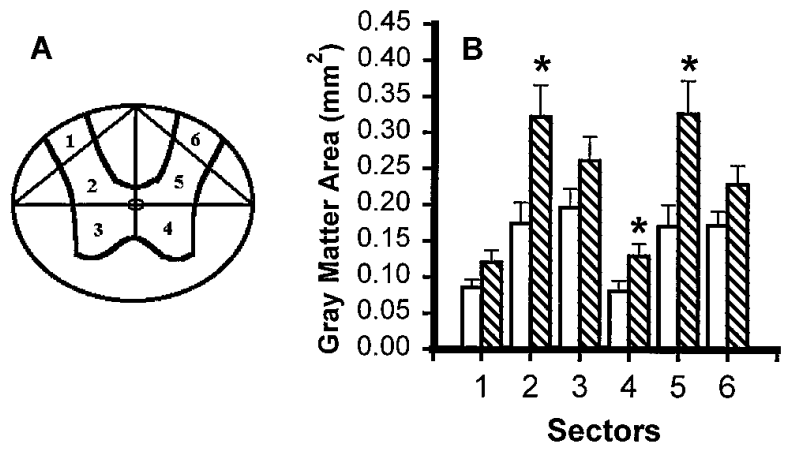

Figure 10. Effect of microinjected TTX on residual GM in different sectors of the spinal cord at 8 weeks after injury. $A$, GM sectors that were analyzed. $B, \mathrm{GM}$ sector analysis of sections at $3 \mathrm{~mm}$ caudal to the lesion epicenter in groups of rats microinjected with VEH alone (open bars) or $0.15 \mathrm{nmol}$ TTX (hatched bars) at $15 \mathrm{~min}$ after SCI ( $n=12$ per group). An overall significant difference $(p<0.05)$ was found between the groups. Asterisks indicate that means are significantly different from the VEHtreated group in the specified sectors (Tukey's procedure).

this possibility. Our finding of significant sparing of WM in the chronic injury site at 8 weeks p.i., which was correlated to reduced functional deficits and associated with improved innervation caudal to the injury by brainstem serotonergic fibers, indicates that TTX is highly protective of WM in vivo. Hence, the present results are consistent with the hypothesis that $\mathrm{Na}^{+}$channels play a significant role in secondary injury to WM that occurs after SCI and contributes to long-term functional impairment.

An alternative explanation could be based on reports showing that $\mathrm{Na}^{+}$channel blockers such as TTX can rescue neuronal cell bodies from anoxic insult through reducing release of glutamate and aspartate: in other words, by preventing excitotoxicity (Leach et al., 1993; Lysko et al., 1994; Lynch et al., 1995; Okiyama et al., 1995). This alternative hypothesis, however, cannot adequately explain our results. With TTX we observed a preferential sparing of WM as compared with GM, the opposite of what would be expected based on an excitotoxic mechanism. Furthermore, in comparing our present results with those obtained when an optimal dose of the glutamate receptor antagonist 2,3-dihydroxy-6nitro-7-sulfamoyl-benzo $(f)$ quinoxaline was microinjected into the injury site at 15 min p.i. (Wrathall et al., 1994), there was a relatively greater effect of TTX in sparing WM and a lesser effect in sparing GM. Thus, although TTX may also reduce neuronal loss by blocking excitotoxicity, as has been postulated for TTX and other $\mathrm{Na}^{+}$channel blockers used in studies of experimental brain injury (Leach et al., 1993; Lysko et al., 1994; Sun and Faden, 1995), we postulate that the main effect of the use of TTX in SCI is through a direct reduction of WM pathology. Ultrastructural studies are in progress to further investigate this hypothesis, and preliminary results indicate a reduction in acute axonal pathology with TTX (Rosenberg et al., 1996). There could also be an effect of TTX on later WM injury processes, such as apoptotic demyelination (Crowe et al., 1997).

Our results with TTX in vivo are consistent with findings from two in vitro models that have been used to study mechanisms of WM injury. In these models TTX was shown to ameliorate the effects of anoxia (Stys et al., 1992b; Stys, 1995) and mechanical compression (Agrawal and Fehlings, 1996) in reducing axonal function, i.e., compound action potential. However, the use of in vitro models precluded the possibility of any histological proof of long-term axonal sparing that results from blocking $\mathrm{Na}^{+}$influx, or 
evidence of effect in reducing long-term functional impairment. Furthermore, for the purpose of studying mechanisms, the drugs tested in these models were applied between 15 and 60 min before the insults (Stys et al., 1992a; Fern et al., 1993; Stys, 1995; Agrawal and Fehlings, 1996), leaving open the question of how "secondary" the $\mathrm{Na}^{+}$-mediated events might be in causing WM loss after traumatic SCI. The current results strongly support the concept that delayed axonal injury that is secondary to the initial mechanical injury of SCI is mediated, at least in part, by $\mathrm{Na}^{+}$channels in the WM. Moreover, this postulated secondary axonal injury seems to contribute significantly to loss of WM and permanent functional impairments.

The pathophysiology of traumatic SCI is understood to be based on primary mechanical injury to the cord tissue and delayed secondary injury processes manifested by the slow development of histopathological changes and the lengthy period required for stabilization of the histological lesions and functional deficits (Allen, 1914; Balentine, 1978a,b; Bresnahan, 1978; Noble and Wrathall, 1989a). A number of mechanisms are involved in the secondary injury process, e.g., ischemia, abnormal ionic shifts across cell membranes, including shifts of $\mathrm{Na}^{+}$and $\mathrm{Ca}^{2+}$, free radical-mediated lipid peroxidation of cell membranes, and the abnormal release of excitatory amino acid neurotransmitters with consequent excitotoxicity (Banik et al., 1987; Saunders and Horrocks, 1987; Faden and Simon, 1988; Tator and Fehlings, 1991; Young, 1993). Our current knowledge of secondary injury processes is based largely on studies focused on damage to neuronal cell bodies (e.g., Regan and Choi, 1991). Much less has been learned about mechanisms of secondary injury to axons in the spinal WM.

Degeneration of tissue after SCI develops later and more slowly in WM than in GM (Balentine, 1978a,b). It involves the accumulation of calcium in the axoplasm (Balentine and Spector, 1977) that is believed to activate proteases and trigger collapse of the axonal cytoskeleton (Kampfl et al., 1996; Saatman et al., 1996). However, although calcium accumulation in neuronal cell bodies after injury seems largely mediated by receptors for excitatory amino acid neurotransmitters (Meldrum and Garthwaite, 1990), the absence of such receptor-coupled and voltage-gated $\mathrm{Ca}^{2+}$ channels on axons requires a different explanation for axoplasmic $\mathrm{Ca}^{2+}$ accumulation after SCI (Waxman, 1991; Stys et al., 1992b).

The recent in vitro studies have provided new and testable hypotheses for events in WM injury that could lead to eventual axonal degeneration. In anoxic injury to the optic nerve in vitro, it is clear that abnormal $\mathrm{Ca}^{2+}$ influx occurs through "reverse operation" of the $\mathrm{Na}^{+}-\mathrm{Ca}^{2+}$ exchanger (Stys et al., 1991b). In the spinal WM compression injury model (Agrawal and Fehlings, 1996), however, blockade of the $\mathrm{Na}^{+}-\mathrm{H}^{+}$exchanger was protective; blockade of the $\mathrm{Na}^{+}-\mathrm{Ca}^{2+}$ exchanger was not protective. The role of $\mathrm{Ca}^{2+}$ in this mechanical axonal injury model was not described. Nevertheless, in both in vitro models, the protective effect of blocking $\mathrm{Na}^{+}$channels is clear.

Because ischemia and resultant anoxia develop over a period of hours in the WM after SCI (Sandler and Tator, 1972; Young, 1985), anoxia-dependent secondary axonal injury is an attractive therapeutic target. Although TTX itself is not likely to be clinically useful because of its severe toxicity, it provides an excellent tool for experimental studies. In the future, dose-response and timecourse studies with TTX could be used to determine lower doses that are protective of axons but do not block action potentials and the temporal window of opportunity for use of other, safer $\mathrm{Na}^{+}$ channel blockers, especially drugs that selectively block slowinactivating $\mathrm{Na}^{+}$channels (Stys et al., 1992a). A recent report on mexiletine, a drug belonging to the category of use-dependent $\mathrm{Na}^{+}$channel blockers, strongly indicates the feasibility and validity of this approach (Stys and Lesiuk, 1996).

There are two previously published studies on the use of the $\mathrm{Na}^{+}$channel blocker lidocaine in experimental SCI. One reported a beneficial effect (Kobrine et al., 1984) and the second (Haghighi et al., 1987) reported no discernible effect. In these studies in which lidocaine was administered intravenously, plasma concentrations were approximately an order of magnitude lower than concentrations found to be protective in the recent in vitro studies (Stys et al., 1992a; Agrawal and Fehlings, 1996). Moreover, in both of these early studies, the outcome measured was restricted to acute (up to $4 \mathrm{hr}$ after SCI) somatosensory evoked potentials. More studies are needed to further explore the effects of $\mathrm{Na}^{+}$ channel blockers on experimental SCI in vivo.

In addition, drugs blocking mechanisms postulated to be downstream of the $\mathrm{Na}^{+}$influx in the process of axonal injury, such as the $\mathrm{Na}^{+}-\mathrm{Ca}^{2+}$ exchanger (Stys et al., 1992b) and the $\mathrm{Na}^{+}-\mathrm{H}^{+}$exchanger (Agrawal and Fehlings, 1996), are potential therapeutic agents. We are currently examining the time course of development of axonal pathology in our model of SCI and the pathophysiological role of these exchangers. Such studies on mechanisms of axonal pathology in SCI will provide the basis for therapies focused specifically on reducing secondary injury to axons and WM.

In conclusion, the results of our current study strongly suggest that axonal $\mathrm{Na}^{+}$channels are causally involved in secondary injury to axons after SCI, with consequent loss of WM at the injury site. The strong correlation seen between WM preservation and reduced chronic functional impairment further supports the hypothesis that such secondary WM injury contributes significantly to the deleterious consequences of SCI. Thus, treatment of acute SCI with drugs that block $\mathrm{Na}^{+}$channels, as well as downstream events in axonal pathology, could form the basis of a new therapeutic approach for SCI.

\section{REFERENCES}

Agrawal SK, Fehlings MG (1996) Mechanisms of secondary injury to spinal cord axons in vitro: role of $\mathrm{Na}^{+}, \mathrm{Na}^{+}-\mathrm{K}^{+}$-ATPase, the $\mathrm{Na}^{+}-\mathrm{H}^{+}$ exchanger, and the $\mathrm{Na}^{+}-\mathrm{Ca}^{2+}$ exchanger. J Neurosci 16:545-552.

Allen AR (1914) Remarks on histopathological changes in spinal cord due to impact: an experimental study. J Nerv Ment Dis 41:141-147.

Balentine JD (1978a) Pathology of experimental spinal cord trauma. I. The necrotic lesion as a function of vascular injury. Lab Invest $39: 236-253$.

Balentine JD (1978b) Pathology of experimental spinal cord trauma. II. Ultrastructure of axons and myelin. Lab Invest 39:254-266.

Balentine JD, Spector M (1977) Calcification of axons in spinal cord trauma. Ann Neurol 2:520-523.

Banik NL, Hogan EL, Hsu CY (1987) The multimolecular cascade of spinal cord injury: studies on prostanoids, calcium, and proteinases. Neurochem Pathol 7:57-77.

Basso DM, Beattie MS, Bresnahan JC (1995) A sensitive and reliable locomotor rating scale for open field testing in rats. J Neurotrauma 12:1-21.

Blight AR (1983) Cellular morphology of chronic spinal cord injury in the cat: analysis of myelinated axons by line sampling. Neuroscience 10:521-543.

Blight AR, Young W (1990) Axonal morphometric correlates of evoked potentials in experimental spinal cord injury. In: Neural monitoring (Salzman S, ed), pp 83-113. New York: Humana.

Bresnahan JC (1978) An electron microscopic analysis of axonal alterations following blunt contusion of the spinal cord of the Rhesus monkey (Macaca mulatta). J Neurol Sci 37:59-82.

Carmignoto G, Vicini S (1992) Activity-dependent decrease in NMDA receptor responses during development of the visual cortex. Science 258:1007-1011. 
Crowe MJ, Bresnahan JC, Shuman SL, Masters JN, Beattie MS (1997) Apoptosis and delayed degeneration after spinal cord injury in rats and monkeys. Nat Med 3:73-76.

Dohrmann GJ, Wagner FC, Bucy PC (1972) Transitory traumatic paraplegia: electron microscopy of early alterations in myelinated nerve fibers. J Neurosurg 36:407-415.

Faden AI, Simon RP (1988) A potential role for excitotoxins in the pathophysiology of spinal cord injury. Ann Neurol 23:623-626.

Faden AI, Gannon AL, Basbaum AI (1988) Use of serotonin immunocytochemistry as a marker of injury severity after experimental spinal trauma in rats. Brain Res 450:94-100.

Fern R, Ransom BR, Stys PK, Waxman SG (1993) Pharmacological protection of CNS white matter during anoxia: actions of phenytoin carbamazepine and diazepam. J Pharmacol Exp Ther 266:1549-1555.

Gale K, Kerasidis H, Wrathall JR (1985) Spinal cord contusion in the rat: behavioral analysis of functional neurological impairment. Exp. Neurol. 88:123-134.

Guth L, Albuquerque EX, Deshpande SS, Barrett CP, Donati EJ, Warnick JE (1980) Ineffectiveness of enzyme therapy on regeneration in the transected spinal cord of the rat. J Neurosurg 52:73-86.

Haghighi SS, Chehrazi BB, Higgins RS, Remington WJ, Wagner FC (1987) Effect of lidocaine treatment on acute spinal cord injury. Neurosurgery 20:536-541.

Hille B (1992) Ionic channels of excitable membranes. Sunderland, MA: Sinauer.

Kampfl A, Posmantur R, Nixon R, Grynspan F, Zhao X, Liu SJ, Newcomb JK, Clifton GL, Hayes RL (1996) Mu-calpain activation and calpainmediated cytoskeletal proteolysis following traumatic brain injury. J Neurochem 67:1575-1583.

Kerasidis H, Wrathall JR, Gale K (1987) Behavioral assessment of functional deficit in rats with contusive spinal cord injury. J Neurosci Methods 20:167-189.

Kobrine AI, Evans DE, LeGrys DC, Yaffe LJ, Bradley ME (1984) Effect of intravenous lidocaine on experimental spinal cord injury. J Neurosurg 60:595-601.

Koo EH, Hoffman PN, Price DL (1988) Levels of neurotransmitter and cytoskeletal protein mRNAs during nerve regeneration in sympathetic ganglia. Brain Res 449:361-363.

Lampert PW (1967) A comparative electron microscopic study of reactive, degenerating, regenerating, and dystrophic axons. J Neuropathol Exp Neurol 26:345-368

Leach MJ, Swan JH, Eisenthal D, Dopson M, Nobbs M (1993) BW619C89, a glutamate release inhibitor, protects against focal cerebral ischemic damage. Stroke 24:1063-1067.

Lynch III JJ, Yu SP, Canzoniero LM, Sensi SL, Choi DW (1995) Sodium channel blockers reduce oxygen-glucose deprivation-induced cortical neuronal injury when combined with glutamate receptor antagonists. J Pharmacol Exp Ther 273:554-560.

Lysko PG, Web CL, Yue TL, Feuerstein G (1994) Neuroprotective effects of tetrodotoxin as a $\mathrm{Na}^{+}$channel modulator and glutamate release inhibitor in cultured rat cerebellar neurons and in gerbil global brain ischemia. Stroke 25:2476-2482.

Meldrum B, Garthwaite J (1990) Excitatory amino acid neurotoxicity and neurodegenerative disease. Trends Pharmacol Sci 11:379-387.

Newman EA (1993) Inward-rectifying potassium channels in retinal glial (Müller) cells. J Neurosci 13:3333-3345.

Noble LJ, Wrathall JR (1985) Spinal cord contusion in the rat: morphometric analyses of alterations in the spinal cord. Exp Neurol 88:135-149.

Noble LJ, Wrathall JR (1989a) Correlative analysis of lesion development and functional status after graded spinal cord contusive injuries in the rat. Exp Neurol 103:34-40.

Noble LJ, Wrathall JR (1989b) Distribution and time course of protein extravasation in the spinal cord after contusive injury. Brain Res 482:57-66.

Okiyama K, Smith DH, Gennarelli TA, Simon RP, Leach M, McIntosh TK (1995) The sodium channel blocker and glutamate release inhibitor BW1003C87 and magnesium attenuate regional cerebral edema following experimental brain injury in the rat. J Neurochem 64:802-809.

Orkand RK (1977) Glial cells. In: Handbook of physiology, the nervous system, cellular biology of neurons (Brookhardt J, Mountcastle V, eds), pp 855-875. Washington, DC: American Physiological Society.

Panjabi M, Wrathall JR (1988) Biomechanical analysis of spinal cord injury and functional loss. Spine 13:1365-1370.

Raines A, Dretchen KL, Marx K, Wrathall JR (1988) Spinal cord contusion in the rat: somatosensory evoked potentials as a function of graded injury. J Neurotrauma 5:151-160.
Ransom BR, Stys PK, Waxman SG (1990a) The pathophysiology of anoxia in mammalian white matter. Stroke [Suppl] 21:III52-III57.

Ransom BR, Waxman SG, Davis PK (1990b) Anoxic injury of CNS white matter: protective effect of ketamine. Neurology 40:1399-1403.

Regan RF, Choi DW (1991) Glutamate neurotoxicity in spinal cord cell culture. Neuroscience 43:585-591.

Rosenberg LJ, Teng YD, Wrathall JR (1996) Assessment of acute axonal pathology after spinal cord injury (abstract). J Neurotrauma 13:604.

Saatman KE, Murai H, Bartus RT, Smith DH, Hayward NJ, Perri BR, McIntosh TK (1996) Calpain inhibitor AK295 attenuates motor and cognitive deficits following experimental brain injury in the rat. Proc Natl Acad Sci USA 93:3428-3433.

Sandler AN, Tator CH (1972) Review of the effects of spinal cord trauma on vessels and blood flow in the spinal cord. J Neurosurg 45:638-646.

Saunders R, Horrocks LA (1987) Eicosanoids, plasma membranes, and molecular mechanisms of spinal cord injury. Neurochem Pathol 7:57-77.

Shapiro SS, Wilk MB (1965) An analysis of variance test of normality. Biometrika 52:591-612.

Skagerberg G, Bjorklund A (1985) Topographic principles in the spinal projections of serotonergic and non-serotonergic brainstem neurons in the rat. Neuroscience 15:445-480.

Sontheimer H, Fernandez-Marques E, Ullrich N, Pappas CA, Waxman SG (1994) Astrocyte $\mathrm{Na}^{+}$channels are required for maintenance of $\mathrm{Na}^{+} / \mathrm{K}^{+}$-ATPase activity. J Neurosci 14:2464-2475.

Stys PK (1995) Protective effects of antiarrhythmic agents against anoxic injury in CNS white matter. J Cereb Blood Flow Metab 15:425-432.

Stys PK, Lesiuk H (1996) Correlation between electrophysiological effects of mexiletine and ischemic protection in central nervous system white matter. Neuroscience 71:27-36.

Stys PK, Ransom BR, Waxman SG, Davis PK (1990) Role of extracellular calcium in anoxic injury of mammalian central white matter. Proc Natl Acad Sci USA 87:4212-4216.

Stys PK, Ransom BR, Waxman SG (1991a) Compound action potential of nerve recorded by suction electrode: a theoretical and experimental analysis. Brain Res 546:18-32.

Stys PK, Waxman SG, Ransom BR (1991b) $\mathrm{Na}^{+}-\mathrm{Ca}^{2+}$ exchanger mediates $\mathrm{Ca}^{2+}$ influx during anoxia in mammalian central nervous system white matter. Ann Neurol 30:375-380.

Stys PK, Ransom BR, Waxman SG (1992a) Tertiary and quaternary local anesthetics protect CNS white matter from anoxic injury at concentrations that do not block excitability. J Neurophysiol 67:236-240.

Stys PK, Waxman SG, Ransom BR (1992b) Ionic mechanisms of anoxic injury in mammalian CNS white matter: role of $\mathrm{Na}^{+}$channels and $\mathrm{Na}^{+}-\mathrm{Ca}^{2+}$ exchanger. J Neurosci 12:430-439.

Sun F, Faden AI (1995) Neuroprotective effects of 619C89, a usedependent sodium channel blocker, in rat traumatic brain injury. Brain Res 673:133-140.

Tator CH, Fehlings MG (1991) Review of the secondary injury theory of acute spinal cord trauma with emphasis on vascular mechanisms. J Neurosurg 75:15-26.

Teng YD, Wrathall JR (1996) Evaluation of cardiorespiratory parameters in rats after spinal cord trauma and treatment with NBQX, an antagonist of excitatory amino acid receptors. Neurosci Lett 209:5-8.

Tork I (1990) Anatomy of the serotonergic system. Ann NY Acad Sci 600:9-35.

Waxman SG (1991) Nonsynaptic mechanisms of calcium-mediated injury in CNS white matter. Trends Neurosci 14:461-468.

Windle WF, Smart JO, Beers JJ (1958) Residual function after subtotal spinal cord transection in adult cats. Neurology 8:518-521.

Wrathall JR, Pettegrew R, Harvey F (1985) Spinal cord contusion in the rat: production of graded, reproducible injury groups. Exp Neurol 88:108-122.

Wrathall JR, Teng YD, Choiniere D, Mundt D (1992) Evidence that local non-NMDA receptors contribute to functional deficits in contusive spinal cord injury. Brain Res 586:140-143.

Wrathall JR, Choiniere D, Teng YD (1994) Dose-dependent reduction of tissue loss and functional impairment after spinal cord trauma with the AMPA/kainate antagonist NBQX. J Neurosci 14:6598-6607.

Young W (1985) Blood flow metabolic and neurophysiologic mechanisms in spinal cord injury. In: Central nervous system trauma status report (Becker D, Povlishock JT, eds), pp 463-473. Bethesda, MD: NIH, NINCDS

Young W (1993) Secondary injury mechanisms in acute spinal cord injury. J Emerg Med 11:13-22. 